\title{
Stabilization of a Class of Stochastic Nonlinear Systems
}

\author{
Valiollah Ghaffari, ${ }^{1}$ Hamid Reza Karimi, ${ }^{2}$ Navid Noroozi, ${ }^{3}$ and S. Vahid Naghavi ${ }^{4}$ \\ ${ }^{1}$ School of Electrical and Computer Engineering, Shiraz University, 7134851151 Shiraz, Iran \\ ${ }^{2}$ Department of Engineering, Faculty of Engineering and Science, University of Agder, 4898 Grimstad, Norway \\ ${ }^{3}$ Young Researchers and Elites Club, Najaf Abad Branch, Islamic Azad University, Najaf Abad, Isfahan, Iran \\ ${ }^{4}$ Young Researchers and Elites Club, Zarghan Branch, Islamic Azad University, Zarghan, Iran
}

Correspondence should be addressed to Navid Noroozi; navid.noroozi@ymail.com

Received 8 August 2013; Accepted 20 October 2013

Academic Editor: Xiao He

Copyright ( 2013 Valiollah Ghaffari et al. This is an open access article distributed under the Creative Commons Attribution License, which permits unrestricted use, distribution, and reproduction in any medium, provided the original work is properly cited.

\begin{abstract}
This paper addresses two control schemes for stochastic nonlinear systems. Firstly, an adaptive controller is designed for a class of motion equations. Then, a robust finite-time control scheme is proposed to stabilize a class of nonlinear stochastic systems. The stability of the closed-loop systems is established based on stochastic Lyapunov stability theorems. Links between these two methods are given. The efficiency of the control schemes is evaluated using numerical simulations.
\end{abstract}

\section{Introduction}

There has been conspicuous attention toward extending popular nonlinear control design in deterministic setting to stochastic framework. In particular, the integrator backstepping was generalized for stochastic nonlinear system in [1]. The author in [2] gave an extension of output feedback backstepping design for stochastic systems. Battiloti [3] investigated stabilization for a class of stochastic nonlinear systems in upper triangular. A stochastic version of nonlinear small gain was given in [4]. Using the small gain condition, the authors provided an adaptive backstepping controller. Other attempts toward this end have been reported in [5-10] and references therein.

Asymptotic stabilization is an important issue in many engineering applications. But for very demanding applications, finite-time stabilization offers an effective alternative, which yields, in some sense, fast response, high tracking precision, and disturbance-rejection properties [11]. Finitetime stability $[12,13]$ allows solving the finite-time stabilization problem. Finite-time stabilization method was introduced by Bhat and Bernstein [12] and then it has been developed by many other researchers (see, e.g., $[13,14])$. As mentioned above, over the last few decades, considerable research works have been devoted to analysis and design of nonlinear stochastic systems. Recently, Chen and Jiao [15] have extended finite-time stability of deterministic systems to stochastic framework using the Itô differential equation.

In this paper, we provide two nonlinear control designs with applications to a guidance system in stochastic setting. The former gives an adaptive control law for the guidance system. The latter stabilizes a class of stochastic system in finite-time; as a special case, it is applied to the guidance system. Two numerical simulations illustrate the effectiveness of the proposed control schemes. Moreover, links between these two methods are given in Section 4.

The rest of this paper is organized as follows. In Section 2, notions for stochastic nonlinear systems are reviewed. In Section 3, a Lyapunov-based adaptive stochastic control is presented for the guidance system; then the result is verified with numerical simulations. In Section 4, a finite-time stochastic control is investigated. Using this control method, a robust finite-time guidance law is derived. In Section 5, concluding remarks are placed.

\section{Preliminaries}

List of Properties

(i) $R_{+}$is the nonnegative real numbers.

(ii) $C^{n}$ denotes the set of $n$-times differentiable functions $R^{n}$ to $R_{+}$. 
(iii) $|\mathbf{x}|$ stands for Euclidean norm.

(iv) A function $\alpha: R_{+} \rightarrow R_{+}$is of class- $\mathscr{K}(\alpha \in \mathscr{K})$ if it is continuous, zero at zero and strictly increasing. Furthermore, it is of class- $\mathscr{K}_{\infty}\left(\alpha \in \mathscr{K}_{\infty}\right)$ if $|s| \rightarrow \infty$ as $s \rightarrow \infty$.

(v) A function $\beta: R_{+} \times R_{+} \rightarrow R_{+}$is of class- $\mathscr{K} \mathscr{L}$ if for each fixed $t \geq 0, \beta(\cdot, t) \in \mathscr{K}$ and for each fixed $s \geq 0, \beta(s, \cdot)$ is strictly decreasing and $\beta(s, t) \rightarrow 0$ as $t \rightarrow \infty$.

(vi) $E[\cdot]$ denotes the expectation of stochastic variable $x$.

Consider the following stochastic nonlinear system:

$$
d \mathbf{x}=(\mathbf{f}(\mathbf{x})+\mathbf{g}(\mathbf{x}) u) d t+\mathbf{h}(\mathbf{x}) d \mathbf{w}
$$

where $\mathbf{x}=\left[x_{1}, \ldots, x_{n}\right]^{T} \in R^{n}$ is the state of the system which is assumed to be available for measurement, $u \in R$ is the control input, $t \in R_{+}$is time, $\mathbf{f} \in R^{n}, \mathbf{g} \in R^{n}$, and $\mathbf{h} \in R^{n \times r}$ are continuous functions, and $\mathbf{w}$ is an $r$-dimensional standard Brownian motion. Let $x\left(t, x_{0}\right)$ denote the solution to the system (1) starting from the initial value $x_{0}$. The system (1) can be thought of as a perturbation from the deterministic system $\dot{\mathbf{x}}=\mathbf{f}(\mathbf{x})+\mathbf{g}(\mathbf{x}) u$ by an additive white noise.

Let $V: R^{n} \rightarrow R^{+}$with the property that $V \in C^{2}$. The differential operator $L$ is defined by

$$
L V:=\frac{\partial V}{\partial \mathbf{x}}(\mathbf{f}(\mathbf{x})+\mathbf{g}(\mathbf{x}) u)+\frac{1}{2} \operatorname{Tr}\left\{\mathbf{h}^{T} \frac{\partial^{2} V}{\partial \mathbf{x}^{2}} \mathbf{h}\right\},
$$

where $\operatorname{Tr}\{\cdot\}$ denotes the matrix trace. We borrow some notions on stability of stochastic system from $[4,15]$.

Definition 1 (see [4]). The system (1) is said to be input-tostate practically stable (ISpS) in probability if for any $\varepsilon>0$, there exist some $\beta \in \mathscr{K} \mathscr{L}, \gamma \in \mathscr{K}$, and $\delta>0$ such that the following hold:

$$
\begin{array}{r}
P\left\{|x| \leq \beta\left(\left|x_{0}\right|, t\right)+\gamma(|u|)+\delta\right\} \geq 1-\varepsilon, \\
\forall t \geq 0, \quad x_{0} \in R^{n} \backslash\{0\} .
\end{array}
$$

Definition 2 (see [15]). For system (1), define $T\left(x_{0}, w\right)=\{T \geq$ $\left.0: x\left(t, x_{0}\right) \equiv 0 \forall t>T\right\}$, which is called the stochastic settling time function.

Definition 3 (see [15]). For stochastic system (1), the origin $\mathbf{x}=0$ is said to be globally stochastically finite-time stable, if for each $x_{0} \in R^{n}$, the following conditions hold:

(i) stochastic settling time function $T\left(x_{0}, w\right)$ exists with probability one;

(ii) if $T\left(x_{0}, w\right)$ exists, then $E\left[T\left(x_{0}, w\right)\right]<+\infty$;

(iii) the origin is stable.

Theorem 4 (see [4]). For system (1), there exist $V: R^{n} \rightarrow R_{+}$ function with the property that $V \in C^{2}$, functions $\alpha, \underline{\alpha}, \bar{\alpha} \in$ $\mathscr{K}_{\infty}$ and $\gamma \in \mathscr{K}$, and $\delta>0$ such that the following hold:

$$
\begin{gathered}
\underline{\alpha}(\mathbf{x}) \leq V(\mathbf{x}) \leq \bar{\alpha}(\mathbf{x}), \\
L V \leq-\alpha(|\mathbf{x}|)+\gamma(|\mathbf{x}|)+\delta ;
\end{gathered}
$$

then system (1) is ISpS in probability sense. Such a function $V$ is called an ISpS Lyapunov function for (1).

Theorem 5 (see [15]). Consider system (1) with $u=0$. If there exist a positive definite, twice continuously differentiable, and radially unbounded Lyapunov function $V: R^{n} \rightarrow R_{+}$and real numbers $\beta>0$ and $0<\alpha<1$, such that

$$
L V(\mathbf{x}) \leq-\beta[V(\mathbf{x})]^{\alpha},
$$

then the origin of system (1) is globally stochastically finite-time stable.

Lemma 6 (Young's inequality). For any $(a, b) \in R \times R$, for all $(p, q) \in R_{+} \times R_{+}$with $(1 / p)+(1 / q)=1$, and for each $\varepsilon>0$, the following holds:

$$
a b \leq \frac{\varepsilon^{p}}{p}|a|^{p}+\frac{1}{\varepsilon^{q} q}|b|^{q} .
$$

\section{Adaptive Control Design for a Guidance System}

In this section, we design an adaptive control law for the guidance system below under the presence of noise. The geometry of planar interception is shown in Figure 1. The two-dimensional motion equation of the planar interception is [16]

$$
\begin{aligned}
\dot{r} & =V_{T} \cos \left(q-\varphi_{T}\right)-V_{M} \cos \left(q-\varphi_{M}\right), \\
r \dot{q} & =-V_{T} \sin \left(q-\varphi_{T}\right)+V_{M} \sin \left(q-\varphi_{M}\right),
\end{aligned}
$$

where $r$ is the relative range, $q$ is LOS angle, $\varphi_{M}$ and $\varphi_{T}$ are flight-path angle for missile and target, and $V_{M}$ and $V_{T}$ are missile velocity and target velocity, respectively. Differentiating (7) with respect to time yields [16]:

$$
\begin{gathered}
\ddot{r}=r \dot{q}^{2}+w-u, \\
\ddot{q}=-\frac{2 \dot{r}}{r} \dot{q}+\frac{1}{r} w-\frac{1}{r} u,
\end{gathered}
$$

where $u$ and $w$ are missile acceleration normal to line of sight and target acceleration normal to line of sight, respectively. Let $u$ be the control input. Also, define $x(t):=\dot{q}(t)$ for all $t \geq 0$. So we get

$$
\dot{x}=-\frac{2 \dot{r}}{r} x+\frac{1}{r} w-\frac{1}{r} u .
$$

Assumption 7. Suppose that $\dot{r}(t)$ and $w(t)$ are stochastic processes defined by

$$
\begin{aligned}
\dot{r}(t) & =\dot{\bar{r}}(t)+\zeta(t), \\
w(t) & =\bar{w}(t)+\zeta(t),
\end{aligned}
$$

where $\dot{\bar{r}}(t)$ and $\bar{w}(t)$ are deterministic and $\zeta(t)$ is white noise degrading measurements. Using Assumption 7, the system (9) can be represented as

$$
d x=\left(-\frac{2 \dot{\bar{r}}}{r} x+\frac{1}{r} \bar{w}-\frac{1}{r} u\right) d t-\frac{1}{r}(2 x-1) d w .
$$




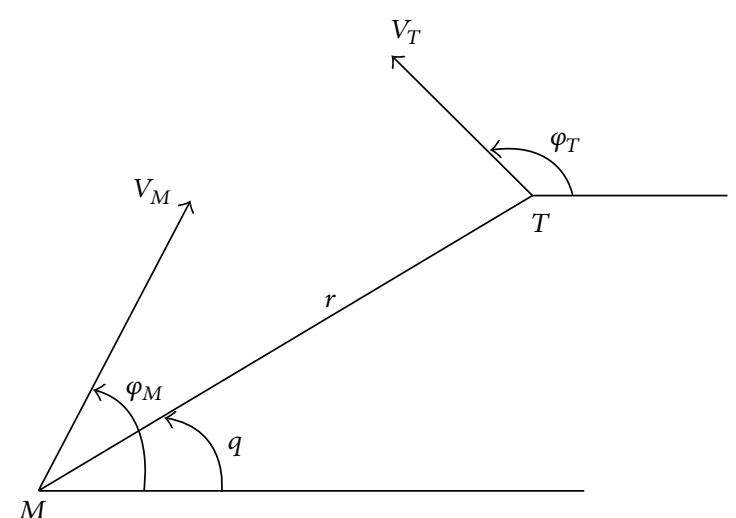

FIgURE 1: Planar interception geometry. $M$ and $T$ denote the missile and the target, respectively.

Theorem 8. Consider the stochastic system (11). The following guidance law

$$
u=c x+\bar{w}-N \dot{\bar{r}} x+\widehat{\theta} \frac{x}{r}+6 \frac{x}{r}-\frac{6}{r}, \quad N>2
$$

with the update law

$$
\dot{\hat{\theta}}=k\left[\sigma\left(\theta^{\circ}-\widehat{\theta}\right)+\frac{x^{4}}{r^{2}}\right] \quad c, \sigma, k>0, \theta^{\circ} \in R
$$

guarantees the boundedness of $\mathbf{x}(t)$ and $\hat{\theta}(t)$ and the convergence of $\mathbf{x}(t)$ to an arbitrary small neighborhood of the origin.

Proof. Define the following Lyapunov function:

$$
V(x, \widehat{\theta})=\frac{1}{4} x^{4}+\frac{1}{2 k} \widetilde{\theta}^{2},
$$

where $\widetilde{\theta}=\theta-\widehat{\theta}$. According to the Itô differential rule [17], we have

$$
\begin{aligned}
L V= & \frac{\partial V}{\partial x}\left(-\frac{2 \dot{\bar{r}}}{r} x+\frac{1}{r} \bar{w}-\frac{1}{r} u\right) \\
& +\frac{1}{2} \frac{\partial^{2} V}{\partial x^{2}}\left(\frac{2 x-1}{r}\right)^{2}+\frac{\partial V}{\partial \hat{\theta}} \dot{\hat{\theta}} .
\end{aligned}
$$

Adding and subtracting $\sigma \widetilde{\theta}\left(\theta^{\circ}-\widehat{\theta}\right)$ to the right-hand sight of (15) give

$$
\begin{aligned}
L V= & x^{3}\left(-\frac{2 \dot{\bar{r}}}{r} x+\frac{1}{r} \bar{w}-\frac{1}{r} u\right) \\
& +\frac{3}{2}\left(\frac{x}{r}\right)^{2}\left(4 x^{2}-4 x+1\right) \\
& -\frac{1}{k} \tilde{\theta} \dot{\hat{\theta}} \pm \sigma \tilde{\theta}\left(\theta^{\circ}-\hat{\theta}\right) .
\end{aligned}
$$

This can be simplified as

$$
\begin{aligned}
L V= & \frac{x^{3}}{r}\left(-2 \dot{\bar{r}} x+\bar{w}-u+\frac{6 x}{r}-\frac{6}{r}\right) \\
& +\frac{3}{2} \frac{x^{2}}{r^{2}}-\frac{1}{k} \tilde{\theta} \dot{\hat{\theta}} \pm \sigma \tilde{\theta}\left(\theta^{\circ}-\widehat{\theta}\right) .
\end{aligned}
$$

Rewrite (17) as

$$
\begin{aligned}
L V= & \frac{x^{3}}{r}\left(-2 \dot{\bar{r}} x+\bar{w}-u+\frac{6 x}{r}-\frac{6}{r}\right) \\
& +\frac{3}{2} \frac{x^{2}}{r^{2}}-\frac{\tilde{\theta}}{k}\left(\dot{\hat{\theta}}-\sigma k\left(\theta^{\circ}-\widehat{\theta}\right)\right)-\sigma \tilde{\theta}\left(\theta^{\circ}-\widehat{\theta}\right),
\end{aligned}
$$

so we get

$$
\begin{aligned}
L V= & \frac{x^{3}}{r}\left(-2 \dot{\bar{r}} x+\bar{w}-u+\frac{6 x}{r}-\frac{6}{r}+\widehat{\theta} \frac{x}{r}\right) \\
& -\widehat{\theta} \frac{x^{4}}{r^{2}}+\frac{3}{2} \frac{x^{2}}{r^{2}}-\frac{\tilde{\theta}}{k}\left(\dot{\hat{\theta}}-\sigma k\left(\theta^{\circ}-\widehat{\theta}\right)\right)-\sigma \widetilde{\theta}\left(\theta^{\circ}-\widehat{\theta}\right) .
\end{aligned}
$$

Recalling the fact that $\widetilde{\theta}=\theta-\widehat{\theta}$, (19) can be written as

$$
\begin{aligned}
L V= & \frac{x^{3}}{r}\left(-2 \dot{\bar{r}} x+\bar{w}-u+\frac{6 x}{r}-\frac{6}{r}+\widehat{\theta} \frac{x}{r}\right) \\
& +(\widetilde{\theta}-\theta) \frac{x^{4}}{r^{2}}+\frac{3}{2} \frac{x^{2}}{r^{2}}-\frac{\widetilde{\theta}}{k}\left(\dot{\hat{\theta}}-\sigma k\left(\theta^{\circ}-\widehat{\theta}\right)\right) \\
& -\sigma \widetilde{\theta}\left(\theta^{\circ}-\widehat{\theta}\right) .
\end{aligned}
$$

Therefore, we have

$$
\begin{aligned}
L V= & \frac{x^{3}}{r}\left(-2 \dot{\bar{r}} x+\bar{w}-u+\frac{6 x}{r}-\frac{6}{r}+\widehat{\theta} \frac{x}{r}\right) \\
& -\theta \frac{x^{4}}{r^{2}}+\frac{3}{2} \frac{x^{2}}{r^{2}}-\frac{\tilde{\theta}}{k}\left(\dot{\hat{\theta}}-\sigma k\left(\theta^{\circ}-\widehat{\theta}\right)-k \frac{x^{4}}{r^{2}}\right) \\
& -\sigma \tilde{\theta}\left(\theta^{\circ}-\widehat{\theta}\right) .
\end{aligned}
$$

It follows with the following update law

$$
\dot{\hat{\theta}}=k\left[\sigma\left(\theta^{\circ}-\widehat{\theta}\right)+\frac{x^{4}}{r^{2}}\right]
$$

that

$$
\begin{aligned}
L V= & \frac{x^{3}}{r}\left(-2 \dot{\bar{r}} x+\bar{w}-u+\frac{6 x}{r}-\frac{6}{r}+\widehat{\theta} \frac{x}{r}\right) \\
& -\theta \frac{x^{4}}{r^{2}}+\frac{3}{2} \frac{x^{2}}{r^{2}}-\sigma \widetilde{\theta}\left(\theta^{\circ}-\widehat{\theta}\right) .
\end{aligned}
$$

The last term in (23) can be written as

$$
2 \widetilde{\theta}\left(\theta^{\circ}-\widehat{\theta}\right)=\widetilde{\theta}^{2}+\left(\theta^{\circ}-\widehat{\theta}\right)^{2}-\left(\theta^{\circ}-\theta\right)^{2} .
$$

Substituting (24) into (21) gives

$$
\begin{aligned}
L V= & \frac{x^{3}}{r}\left(-2 \dot{\bar{r}} x+\bar{w}-u+\frac{6 x}{r}-\frac{6}{r}+\widehat{\theta} \frac{x}{r}\right) \\
& -\frac{\sigma}{2}\left(\widetilde{\theta}^{2}+\left(\theta^{\circ}-\widehat{\theta}\right)^{2}-\left(\theta^{\circ}-\theta\right)^{2}\right)-\theta \frac{x^{4}}{r^{2}}+\frac{3}{2} \frac{x^{2}}{r^{2}} .
\end{aligned}
$$


Pick the following control law:

$$
u=c x+\bar{w}-N \dot{\bar{r}} x+\widehat{\theta} \frac{x}{r}+6 \frac{x}{r}-\frac{6}{r} \quad N>2 .
$$

So we obtain

$$
\begin{aligned}
L V= & -c \frac{x^{4}}{r}+\frac{\dot{\bar{r}} x^{4}}{r}(N-2)-\theta \frac{x^{4}}{r^{2}}+\frac{3}{2} \frac{x^{2}}{r^{2}} \\
& -\frac{\sigma}{2}\left(\widetilde{\theta}^{2}+\left(\theta^{\circ}-\widehat{\theta}\right)^{2}-\left(\theta^{\circ}-\theta\right)^{2}\right) .
\end{aligned}
$$

For $N>2$, the second term on the right-hand side of (27) is negative (i.e., $\left.\left(\dot{\bar{r}} x^{4} / r\right)(N-2)<0\right)$, thus

$$
\begin{aligned}
L V \leq & -c \frac{x^{4}}{r}-\theta \frac{x^{4}}{r^{2}}+\frac{3}{2} \frac{x^{2}}{r^{2}} \\
& -\frac{\sigma}{2}\left(\widetilde{\theta}^{2}+\left(\theta^{\circ}-\widehat{\theta}\right)^{2}-\left(\theta^{\circ}-\theta\right)^{2}\right) .
\end{aligned}
$$

A simplified but conservative version of (28) is

$$
L V \leq-c \frac{x^{4}}{r}-\theta \frac{x^{4}}{r^{2}}+\frac{3}{2} \frac{x^{2}}{r^{2}}-\frac{\sigma}{2}\left(\widetilde{\theta}^{2}-\left(\theta^{\circ}-\theta\right)^{2}\right) .
$$

The last term in (29) on the right-hand side of (29) is negative (i.e., $\left.-\sigma / 2\left(\theta^{\circ}-\widehat{\theta}\right)^{2}<0\right)$, so

$$
L V \leq-c \frac{x^{4}}{r}-\theta \frac{x^{4}}{r^{2}}+\frac{3}{2} \frac{x^{2}}{r^{2}}-\frac{\sigma}{2} \widetilde{\theta}^{2}+\frac{\sigma}{2}\left(\theta^{\circ}-\theta\right)^{2} .
$$

The second and third terms on the right-hand side of (30) can be written as

$$
\begin{aligned}
-\theta \frac{x^{4}}{r^{2}}+\frac{3}{2} \frac{x^{2}}{r^{2}} & =-\frac{\theta}{r^{2}}\left(x^{4}-\frac{3}{2 \theta} x^{2}\right) \\
& =-\frac{\theta}{r^{2}}\left(x^{4}-\frac{3}{2 \theta} x^{2}+\left(\frac{3}{4 \theta}\right)^{2}-\left(\frac{3}{4 \theta}\right)^{2}\right) \\
& =-\frac{\theta}{r^{2}}\left(x^{2}-\frac{3}{4 \theta}\right)^{2}+\frac{9}{16 \theta r^{2}} .
\end{aligned}
$$

By substituting (31) into (30), we get

$$
L V \leq-c \frac{x^{4}}{r}-\frac{\theta}{r^{2}}\left(x^{2}-\frac{3}{4 \theta}\right)^{2}+\frac{9}{16 \theta r^{2}}-\frac{\sigma}{2} \widetilde{\theta}^{2}+\frac{\sigma}{2}\left(\theta^{\circ}-\theta\right)^{2} .
$$

So

$$
L V \leq-c \frac{x^{4}}{r}-\frac{\sigma}{2} \widetilde{\theta}^{2}+\frac{9}{16 \theta r^{2}}+\frac{\sigma}{2}\left(\theta^{\circ}-\theta\right)^{2} \quad \theta>0 .
$$

Let $\varepsilon=\left(9 / 16 \theta r^{2}\right)+(\sigma / 2)\left(\theta^{\circ}-\theta\right)^{2}$. Substituting (14) into the right-hand side of above inequality gives

$$
L V \leq-c_{0} V+\varepsilon
$$

where $c_{0}=\min \{k \sigma, 4 c / r\}$. Inequality (34) implies that $V$ is an ISpS Lyapunov function. Therefore, it ensures the boundedness of $\mathbf{x}(t)$ and $\widetilde{\theta}(t)$ and convergence of $\mathbf{x}(t)$ to an arbitrary small neighborhood of the origin.
Corollary 9. Under the assumption that $w(t)$ is a pure deterministic variable. The following guidance law

$$
u=c x+w-N \dot{\bar{r}} x+\widehat{\theta} \frac{x}{r}, \quad N>2
$$

with an update law

$$
\dot{\theta}=k \frac{x^{4}}{r^{2}} \quad k>0
$$

guarantees the boundedness of $\mathbf{x}(t)$ and $\widehat{\theta}(t)$ and the convergence of $\mathbf{x}(t)$ to the origin.

Proof. The proof is a simple conclusion of Theorem 8.

Three-Dimensional Case. With the same arguments, the result can be extended to the three-dimensional case. Consider the following stochastic system:

$$
\begin{aligned}
& d x_{1}=\left(-\frac{2 \dot{\bar{r}}}{r} x_{1}+\frac{1}{r} \bar{w}_{1}-\frac{1}{r} u_{1}\right) d t-\frac{1}{r}\left(2 x_{1}-1\right) d w_{1} \\
& d x_{2}=\left(-\frac{2 \dot{\bar{r}}}{r} x_{2}+\frac{1}{r} \bar{w}_{2}-\frac{1}{r} u_{2}\right) d t-\frac{1}{r}\left(2 x_{2}-1\right) d w_{2}
\end{aligned}
$$

where $w_{1}=a_{T \theta}$ and $w_{2}=a_{T \phi}$. The following guidance laws

$$
\begin{aligned}
& u_{1}=c_{1} x+\bar{w}_{1}-N \dot{\bar{r}} x_{1}+\widehat{\theta}_{1} \frac{x_{1}}{r}+6 \frac{x_{1}}{r}-\frac{6}{r}, \quad N>2, \\
& u_{2}=c_{2} x+\bar{w}_{2}-N \dot{\bar{r}} x_{2}+\widehat{\theta}_{2} \frac{x_{2}}{r}+6 \frac{x_{2}}{r}-\frac{6}{r}, \quad N>2
\end{aligned}
$$

with the update laws

$$
\begin{array}{ll}
\dot{\hat{\theta}}_{1}=k_{1}\left[\sigma_{1}\left(\theta_{1}^{\circ}-\hat{\theta}_{1}\right)+\frac{x_{1}^{4}}{r^{2}}\right], & c_{1}, \sigma_{1}, k_{1}>0, \theta_{1}^{\circ} \in R, \\
\dot{\hat{\theta}}_{2}=k_{2}\left[\sigma_{2}\left(\theta_{2}^{\circ}-\hat{\theta}_{2}\right)+\frac{x_{2}^{4}}{r^{2}}\right], & c_{2}, \sigma_{2}, k_{2}>0, \theta_{2}^{\circ} \in R
\end{array}
$$

guarantee the boundedness of $\mathbf{x}(t)$ and the convergence of $\mathbf{x}(t)$ to an arbitrary small neighborhood of the origin.

3.1. Numerical Simulation. For the closed loop system, the mentioned stochastic differential equations governed by this guidance system can be simplified as

$$
\begin{aligned}
d x(t)= & \left(-(N+2) \frac{\dot{\bar{r}}(t)}{r(t)} x(t)-\frac{c+\hat{\theta}(t)}{r(t)} x(t)\right. \\
& \left.+\frac{6}{r^{2}(t)} x(t)-\frac{6}{r^{2}(t)}\right) d t \\
& +\left(\frac{-2}{r(t)} x(t)+\frac{1}{r(t)}\right) d w(t)
\end{aligned}
$$


where $\dot{\hat{\theta}}(t)$ and $r(t)$ are simultaneously solved by the following:

$$
\begin{aligned}
\dot{\hat{\theta}}(t)=k[\sigma & \left.\left(\theta^{\circ}-\widehat{\theta}(t)\right)+\frac{x^{4}(t)}{r(t)}\right] \quad c, \sigma, k>0 \quad \theta^{\circ} \in R, \\
\ddot{r}(t)= & \left(r(t) x(t)-c-N \dot{\bar{r}}(t)-\widehat{\theta}(t)+\frac{6}{r(t)}\right) \\
& \times x(t)+\zeta(t)-\frac{6}{r(t)} .
\end{aligned}
$$

To solve these equations numerically, we follow the methods in $[18,19]$. The guidance law and update law parameters are chosen as $N=2, c=500, \sigma=2, K=5$, and $\theta^{0}=0.2$. Initial conditions of system (11) and adaptive guidance law (13) are selected as $\dot{r}(0)=200, r(0)=1, \widehat{\theta}(0)=0$, and $x(0)=0.1$. Sampling time is set to the value $h=0.005$. Applying our proposed method, the line of sight rate $x(t)$ is depicted in Figure 2.

The update parameter $\hat{\theta}(t)$ is shown in Figure 3. As we expect, after some transient time ( 0.4 second), the update parameter stays close to the value of 0.2 .

The control input $u(t)$ is shown in Figure 4.

\section{Finite-Time Control Law for Stochastic systems}

In the previous section, the variable $\dot{q}$ is only controlled (see (9)). One expects to get better performance of the guidance system if both the LOS angle $q$ and the angular velocity $\dot{q}$ are controlled. In this case, we get the following state-space equations:

$$
\begin{gathered}
\dot{x}_{1}=x_{2}, \\
\dot{x}_{2}=-\frac{2 \dot{r}}{r} x_{2}+\frac{1}{r} w-\frac{1}{r} u .
\end{gathered}
$$

Using Assumption 7, the system (42) can be represented as

$$
\begin{gathered}
d x_{1}=x_{2} d t, \\
d x_{2}=\left(-\frac{2 \dot{\bar{r}}}{r} x_{2}+\frac{1}{r} \bar{w}-\frac{1}{r} u\right) d t-\frac{1}{r}\left(2 x_{2}-1\right) d w .
\end{gathered}
$$

So we aim to develop a guidance law to stabilize the origin of the system (43). On the other hand, the notion of finitetime stability is very important in guidance problems since the guidance equations are valid as long as the intercept point is met in finite-time $[16,20,21]$. The adaptive guidance law proposed in the previous section does not provide the finitetime convergence of the guidance system although it gives the effective robustness. These motivate us to give a robust finitetime guidance law in stochastic setting.

Consider the following stochastic nonlinear system:

$$
\begin{gathered}
d x_{1}=x_{2} d t, \\
d x_{2}=(f(\mathbf{x}, t)+u) d t+g(\mathbf{x}, t) d w,
\end{gathered}
$$

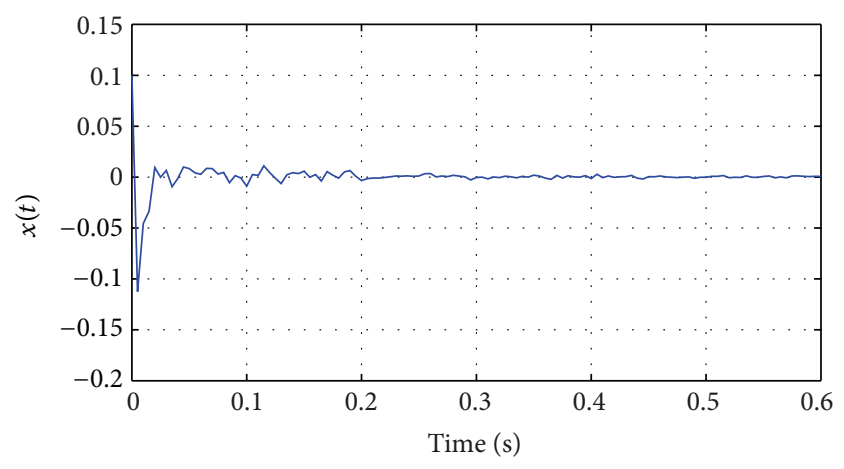

FIGURE 2: The line of sight rate $x(t)$.

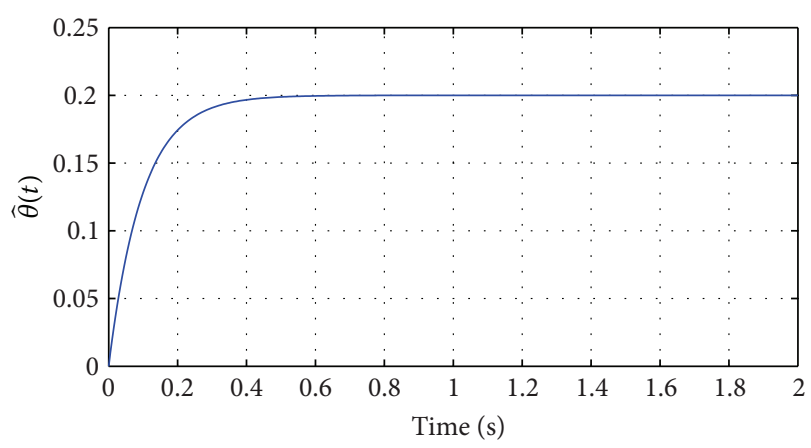

FIgURE 3: The adaptation parameter $\widehat{\theta}(t)$.

where $w \in R$ and $\mathbf{x}=\left[x_{1}, x_{2}\right]^{T} \in R^{2}$ is assumed to be available for measurement. A guidance system can be modeled in this form. We emphasize that the guidance system (43) is a special case of the system (44).

Assumption 10. For any $\mathbf{x} \in R^{2}$, the nonlinear part of (42) can be bounded by

$$
|f(\mathbf{x}, t)| \leq \gamma\left(x_{1}, x_{2}\right)\left(\left|x_{1}\right|+\left|x_{2}\right|\right),
$$

where $\gamma\left(x_{1}, x_{2}\right) \geq 0$ is a known $C^{1}$ function.

It should be noted that the function $f(\cdot, \cdot)$ is unknown, in general. We only need that the upper bound (45) is given.

Theorem 11. Consider the stochastic system (42) with Assumption 10. The following control law

$$
\begin{aligned}
u= & -\beta\left(x_{1}, x_{2}\right)\left(x_{2}^{5 / 3}+0.15 x_{1}\right)^{1 / 5} \\
& -\left[\frac{7}{3} \omega\left(x_{1}, x_{2}\right)+\frac{\delta^{4}}{4} x_{2}^{8 / 3}\right] \xi_{2}^{1 / 5}
\end{aligned}
$$

guarantees the boundedness of $\mathbf{x}(t)$ and the convergence of $\mathbf{x}(t)$ to the origin in finite-time.

Proof. Define the following Lyapunov function:

$$
V(\mathbf{x})=\frac{1}{2} x_{1}^{2}+\int_{x_{2}^{*}}^{x_{2}}\left(s^{5 / 3}-x_{2}^{*^{5 / 3}}\right)^{7 / 5} d s .
$$




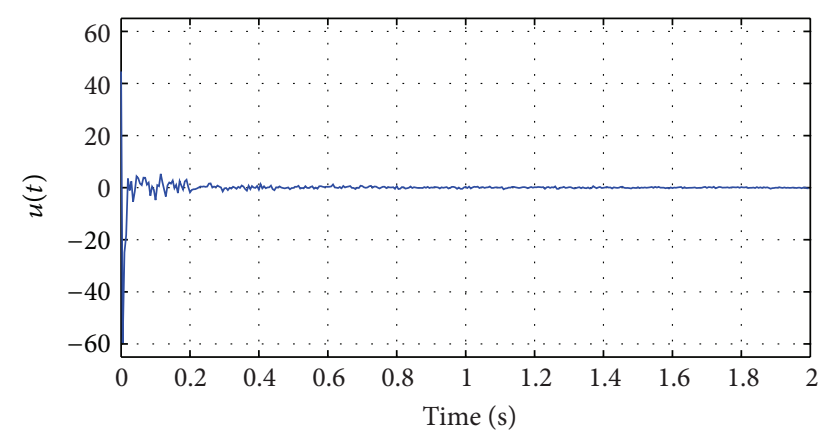

FIGURE 4: The applied control effort.

Fact 1. Recall that for a given dynamical system

$$
d \mathbf{x}=f(\mathbf{x}, t) d t+g(\mathbf{x}, t) d \mathbf{w}(t) .
$$

According to the Itô differential rule $L V(\mathbf{x})$ is

$$
\begin{aligned}
L V(\mathbf{x})= & \frac{\partial V(\mathbf{x})}{\partial \mathbf{x}} f(\mathbf{x}, t) \\
& +\frac{1}{2} \operatorname{Tr}\left(g^{T}(\mathbf{x}, t) \frac{\partial^{2} V(\mathbf{x})}{\partial \mathbf{x}^{2}} g(\mathbf{x}, t)\right) .
\end{aligned}
$$

So $L V(\mathbf{x})$ for the system (42) is

$$
\begin{aligned}
L V(\mathbf{x})= & \frac{\partial V(\mathbf{x})}{\partial x_{1}} x_{2}+\frac{\partial V(\mathbf{x})}{\partial x_{2}}(f(\mathbf{x}, t)+u) \\
& +\frac{1}{2} \frac{\partial^{2} V(\mathbf{x})}{\partial x_{2}^{2}} g^{2}(\mathbf{x}, t) .
\end{aligned}
$$

The partial derivative of $V$ respect to $x_{1}$ is

$$
\frac{\partial V(\mathbf{x})}{\partial x_{1}}=x_{1}+\frac{\partial}{\partial x_{1}} \int_{x_{2}^{*}}^{x_{2}}\left(s^{5 / 3}-x_{2}^{*^{5 / 3}}\right)^{7 / 5} d s .
$$

Fact 2. From the elementary calculus, we get

$$
\begin{aligned}
& \frac{d}{d \mathbf{x}}\left(\int_{a}^{g(\mathbf{x})} f(\mathbf{x}, z) d z\right) \\
& \quad=\int_{a}^{g(\mathbf{x})} \frac{\partial f(\mathbf{x}, z)}{\partial \mathbf{x}} d z+\left.\frac{\partial g(\mathbf{x})}{\partial x} f(\mathbf{x}, z)\right|_{z=g(\mathbf{x})} .
\end{aligned}
$$

Using Fact 2, (49) is rewritten as

$$
\begin{aligned}
\frac{\partial V(\mathbf{x})}{\partial x_{1}}= & x_{1}+\int_{x_{2}^{*}}^{x_{2}} \frac{\partial}{\partial x_{1}}\left(s^{5 / 3}-x_{2}^{*^{5 / 3}}\right)^{7 / 5} d s \\
& -\left.\frac{\partial x_{2}^{*}}{\partial x_{1}}\left(s^{5 / 3}-x_{2}^{*^{5 / 3}}\right)^{7 / 5}\right|_{s=x_{2}^{*}} .
\end{aligned}
$$

It follows with the fact that $\left.\left(\partial x_{2}^{*} / \partial x_{1}\right)\left(s^{5 / 3}-x_{2}^{*^{5 / 3}}\right)^{7 / 5}\right|_{s=x_{2}^{*}}=0$ that

$$
\begin{aligned}
\frac{\partial V(\mathbf{x})}{\partial x_{1}}=x_{1}+\int_{x_{2}^{*}}^{x_{2}} \frac{7}{5} & \left(s^{5 / 3}-x_{2}^{*^{5 / 3}}\right)^{2 / 5} \\
& \times\left(-\frac{5}{3} x_{2}^{*^{2 / 3}}\right) \frac{\partial x_{2}^{*}}{\partial x_{1}} d s .
\end{aligned}
$$

Recall that

$$
x_{2}^{*^{2 / 3}} \frac{\partial x_{2}^{*}}{\partial x_{1}}=\left(-2 x_{1}^{3 / 5}\right)^{2 / 3}\left(-\frac{6}{5} x_{1}^{-2 / 5}\right)=-\frac{6}{5}(4)^{1 / 3}
$$

we obtain

$$
\frac{\partial V(\mathbf{x})}{\partial x_{1}}=x_{1}+\frac{14}{5}(4)^{1 / 3} \int_{x_{2}^{*}}^{x_{2}}\left(s^{5 / 3}-x_{2}^{*^{5 / 3}}\right)^{2 / 5} d s .
$$

The partial derivative of $V(\mathbf{x})$ with respect to $x_{2}$ is

$$
\frac{\partial V(\mathbf{x})}{\partial x_{2}}=\left(x_{2}^{5 / 3}-x_{2}^{*^{5 / 3}}\right)^{7 / 5}=\xi_{2}^{7 / 5}
$$

and the second derivative with respect to $x_{2}$ is

$$
\frac{\partial^{2} V(\mathbf{x})}{\partial x_{2}^{2}}=\frac{7}{3} \xi_{2}^{2 / 5} x_{2}^{2 / 3}
$$

So we have

$$
\begin{aligned}
L V(\mathbf{x})= & \left(x_{1}+\frac{14}{5}(4)^{1 / 3} \int_{x_{2}^{*}}^{x_{2}}\left(s^{5 / 3}-x_{2}^{*^{5 / 3}}\right)^{2 / 5} d s\right) x_{2} \\
& +x_{2}^{7 / 5}(f(\mathbf{x})+u)+\frac{7}{6} x_{2}^{2 / 5} x_{2}^{2 / 3} g^{2}(\mathbf{x}) .
\end{aligned}
$$

Define the following variables:

$$
\xi_{1}:=x_{1}, \quad \xi_{2}:=x_{2}^{5 / 3}-x_{2}^{*^{5 / 3}}, \quad x_{2}^{*}:=-2 x_{1}^{3 / 5} \text {. }
$$

Therefore, one yields:

$$
\begin{aligned}
L V= & \left(\xi_{1}-\frac{7}{5} \int_{x_{2}^{*}}^{x_{2}}\left(s^{5 / 3}-x_{2}^{*^{5 / 3}}\right)^{2 / 5} d s\right) x_{2} \\
& +\xi_{2}^{7 / 5}(f(\mathbf{x})+u)+\frac{7}{3} g(\mathbf{x})^{2} x_{2}^{2 / 3}\left(x_{2}^{5 / 3}-x_{2}^{*^{5 / 3}}\right)^{2 / 5} .
\end{aligned}
$$

Let $u:=u_{d}+u_{s}$; the deterministic term is

$$
u_{d}=-\beta\left(x_{1}, x_{2}\right)\left(x_{2}^{5 / 3}+0.15 x_{1}\right)^{1 / 5} \text {, }
$$

where $\beta\left(x_{1}, x_{2}\right)>0$ is a $C^{1}$ function. Particularly, $u_{d}$ and $u_{s}$ provide the deterministic and stochastic parts of (44), respectively. Substituting (62) into the right-hand side of (61) gives

$$
L V \leq-\left(\xi_{1}^{8 / 5}+\xi_{2}^{8 / 5}\right)+\xi_{2}^{7 / 5} u_{s}+\frac{7}{3} g(\mathbf{x})^{2} x_{2}^{2 / 3} \xi_{2}^{2 / 5} .
$$

Assume that

$$
|g(\mathbf{x}, t)|^{2} \leq \omega\left(x_{1}, x_{2}\right)\left(\left|\xi_{1}\right|^{6 / 5}+\left|\xi_{2}\right|^{6 / 5}\right),
$$

where $\omega\left(x_{1}, x_{2}\right)>0$ is a $C^{1}$ function. From the aforementioned assumption and Young's inequality, we get

$$
\begin{aligned}
L V \leq & -\left(\xi_{1}^{8 / 5}+\xi_{2}^{8 / 5}\right)+\xi_{2}^{7 / 5} u_{s} \\
& +\frac{7}{3} \omega\left(x_{1}, x_{2}\right)\left(\left|\xi_{1}\right|^{6 / 5}+\left|\xi_{2}\right|^{6 / 5}\right) x_{2}^{2 / 3} \xi_{2}^{2 / 5} \\
\leq & -\left(\xi_{1}^{8 / 5}+\xi_{2}^{8 / 5}\right)+\frac{1}{(4 / 3) \delta^{4 / 3}} \xi_{1}^{8 / 5} \\
& +\xi_{2}^{7 / 5}\left(u_{s}+\left[\frac{7}{3} \omega\left(x_{1}, x_{2}\right)+\frac{\delta^{4}}{4} x_{2}^{8 / 3}\right] \xi_{2}^{1 / 5}\right),
\end{aligned}
$$


where $\delta \in(0,1)$. Let $u_{s}$ be

$$
u_{s}=-\left[\frac{7}{3} \omega\left(x_{1}, x_{2}\right)+\frac{\delta^{4}}{4} x_{2}^{8 / 3}\right] \xi_{2}^{1 / 5} \text {. }
$$

With the similar arguments as above, we have

$$
L V \leq-(1-\delta)\left(\xi_{1}^{8 / 5}+\xi_{2}^{8 / 5}\right)
$$

It follows from Proposition 2 and Lemma 2.3 in [13] that

$$
L V \leq-(1-\delta)\left(\xi_{1}^{8 / 5}+\xi_{2}^{8 / 5}\right) \leq-\rho V^{4 / 5} \quad \rho \in(0,0.5)
$$

This shows that the origin of the system is globally stochasticly finite-time stable.

4.1. Numerical Simulation. In this section, the performance of the proposed control schemes is evaluated via a numerical example. Consider the following dynamical system:

$$
\begin{gathered}
d x_{1}=x_{2} d t \\
d x_{2}=u d t+x_{1} d w
\end{gathered}
$$

where $w \approx N(0,0.05)$. Let $\beta\left(x_{1}, x_{2}\right)=0.4$ and $\sigma=0.8$. Initial condition is selected as $x_{1}(0)=1$ and $x_{2}(0)=-1$. A lower bound for $\omega\left(x_{1}, x_{2}\right)$ is

$$
\omega\left(x_{1}, x_{2}\right) \geq \frac{|g(\mathbf{x})|^{2}}{\left|\xi_{1}\right|^{6 / 5}+\left|\xi_{2}\right|^{6 / 5}} .
$$

Therefore, we can choose $\omega\left(x_{1}, x_{2}\right)$ as

$$
\omega\left(x_{1}, x_{2}\right)=b \frac{|g(\mathbf{x})|^{2}}{\left|\xi_{1}\right|^{6 / 5}+\left|\xi_{2}\right|^{6 / 5}},
$$

where $b=1.1$. The control law obtained from the results in Theorem 11 is

$$
\begin{aligned}
u= & -\beta\left(x_{1}, x_{2}\right)\left(x_{2}^{5 / 3}+0.15 x_{1}\right)^{1 / 5} \\
& -\left[\frac{7}{3} \omega\left(x_{1}, x_{2}\right)+\frac{\delta^{4}}{4} x_{2}^{8 / 3}\right] \xi_{2}^{1 / 5},
\end{aligned}
$$

where

$$
\xi_{1}=x_{1}, \quad \xi_{2}=x_{2}^{5 / 3}-x_{2}^{*^{5 / 3}}, \quad x_{2}^{*}=-2 x_{1}^{3 / 5}
$$

Numerical simulations are implemented in MATLAB with the step size 0.001. Figures 5 and 6 illustrate the effectiveness of simulation results using the control law (70). The state trajectories are shown in Figure 5. The control input is depicted in Figure 6. Obviously, the control signal (70) is not smooth although it gives a finite-time convergence.

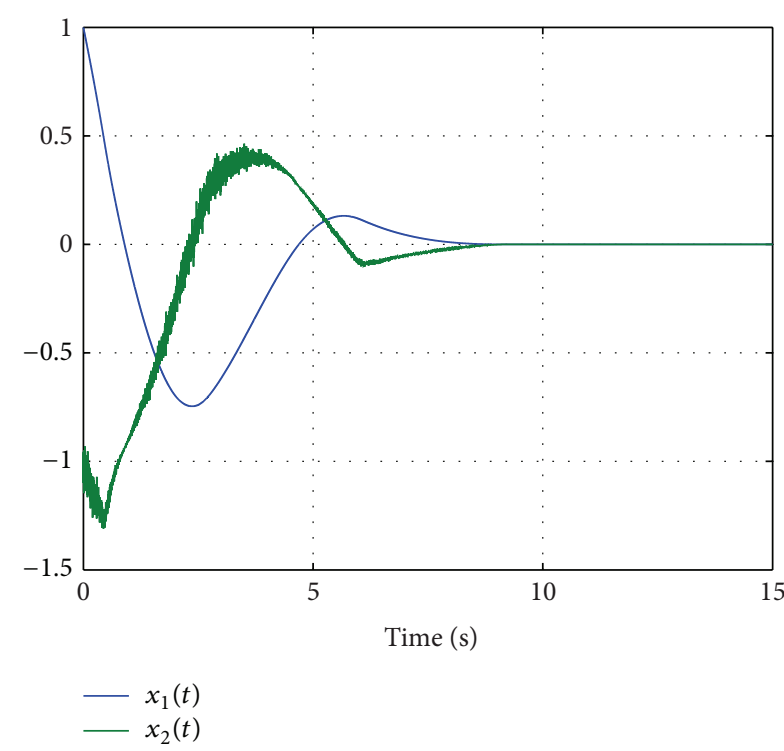

FIgURE 5: The state trajectories $x(t)$ due to the control scheme.

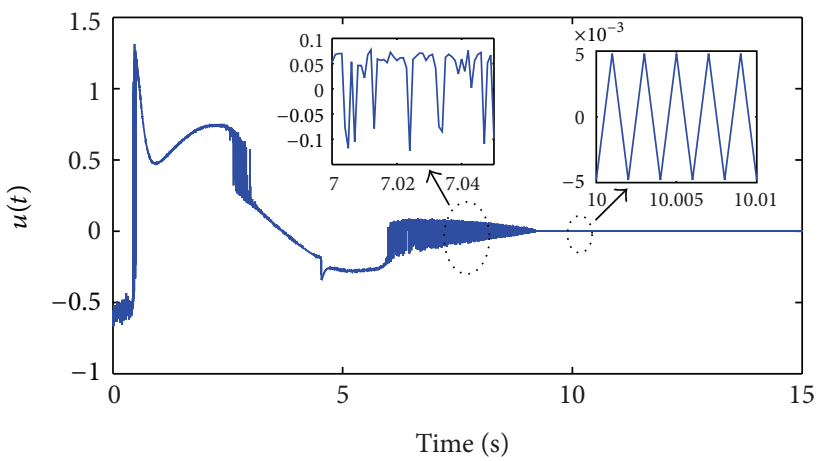

Figure 6: Input control $u(t)$ due to the control scheme.

\section{Conclusions}

Two guidance laws were proposed in stochastic setting. First, an adaptive guidance was presented to achieve target interception under measurement noise. The effectiveness of this result was evaluated using numerical simulations. Although this guidance law provided the effective robustness, it did not guarantee the finite-time convergence for the guidance system which is preferably required. Next, a robust control method was proposed to stabilize both the LOS angle and its angular velocity at the origin in finite-time under measurement noise. Simulation results demonstrated that the second guidance approach provided the finite-time convergence, but the signal input was not as smooth as the one in the first method. So this showed that balance between these approaches was needed.

\section{Conflict of Interests}

The authors declare that there is no conflict of interests regarding the publication of this paper. 


\section{References}

[1] H. Deng and M. Krstić, "Stochastic stabilization part I: a backstepping design," Systems and Control Letters, vol. 32, no. 3, pp. 143-150, 1997.

[2] H. Deng and M. Krstić, "Output-feedback stochastic nonlinear stabilization," IEEE Transactions on Automatic Control, vol. 44, no. 2, pp. 328-333, 1999.

[3] S. Battilotti, "Lyapunov-based design of iISS feedforward systems with uncertainty and noisy measurements," SIAM Journal on Control and Optimization, vol. 46, no. 1, pp. 84-115, 2007.

[4] Z.-J. Wu, X.-J. Xie, and S.-Y. Zhang, "Adaptive backstepping controller design using stochastic small-gain theorem," Automatica, vol. 43, no. 4, pp. 608-620, 2007.

[5] J. Hu, Z. Wang, B. Shen, and H. Gao, "Gain-constrained recursive filtering with stochastic nonlinearities and probabilistic sensor delays," IEEE Transaction on Signal Processing, vol. 61, pp. 1230-1238, 2013.

[6] X. Mu and H. Liu, "Stabilization for a class of stochastic nonlinear systems via output feedback," IEEE Transactions on Automatic Control, vol. 53, no. 1, pp. 360-367, 2008.

[7] Z. Wang, D. W. C. Ho, H. Dong, and H. Gao, "Robust Ho finite-horizon control for a class of stochastic nonlinear timevarying systems subject to sensor and actuator saturations," IEEE Transactions on Automatic Control, vol. 55, no. 7, pp. 17161722, 2010.

[8] Z. Wang, J. Lam, and X. Liu, "Stabilization of a class of stochastic nonlinear time-delay systems," Journal of Nonlinear Dynamics and Systems Theory, vol. 4, pp. 357-368, 2004.

[9] Z. Wu, M. Cui, P. Shi, and H. R. Karimi, "Stability of stochastic nonlinear systems with state-dependent switching," IEEE Transaction on Automatic Control, vol. 58, pp. 1904-1918, 2013.

[10] Y. Yin, P. Shi, and F. Liu, "Gain-scheduled PI tracking control on stochastic nonlinear systems with partially known transition probabilities," Journal of the Franklin Institute, vol. 348, no. 4, pp. 685-702, 2011.

[11] E. Moulay and W. Perruquetti, "Finite time stability and stabilization of a class of continuous systems," Journal of Mathematical Analysis and Applications, vol. 323, no. 2, pp. 1430-1443, 2006.

[12] S. P. Bhat and D. S. Bernstein, "Finite-time stability of continuous autonomous systems," SIAM Journal on Control and Optimization, vol. 38, no. 3, pp. 751-766, 2000.

[13] X. Huang, W. Lin, and B. Yang, "Global finite-time stabilization of a class of uncertain nonlinear systems," Automatica, vol. 41, no. 5, pp. 881-888, 2005.

[14] Y. Orlov, "Finite time stability and robust control synthesis of uncertain switched systems," SIAM Journal on Control and Optimization, vol. 43, no. 4, pp. 1253-1271, 2005.

[15] W. Chen and L. C. Jiao, "Finite-time stability theorem of stochastic nonlinear systems," Automatica, vol. 46, no. 12, pp. 2105-2108, 2010.

[16] D. Zhou, S. Sun, and K. L. Teo, "Guidance laws with finite time convergence," Journal of Guidance, Control, and Dynamics, vol. 32, no. 6, pp. 1838-1846, 2009.

[17] R. Z. Khas'minskii, Stochastic Stability of Differential Equations, S \& N International, Rockville, Md, USA, 1980.

[18] H. Gilsing and T. Shardlow, "SDELab: a package for solving stochastic differential equations in MATLAB," Journal of Computational and Applied Mathematics, vol. 205, no. 2, pp. 10021018, 2007.
[19] S. Rubenthaler, "Numerical simulation of the solution of a stochastic differential equation driven by a Lévy process," Stochastic Processes and Their Applications, vol. 103, no. 2, pp. 311-349, 2003.

[20] S. Sun, D. Zhou, and W.-T. Hou, "A guidance law with finite time convergence accounting for autopilot lag," Aerospace Science and Technology, vol. 25, pp. 132-137, 2013.

[21] Y. Zhang, M. Sun, and Z. Chen, "Finite-time convergent guidance law with impact angle constraint based on slidingmode control," Nonlinear Dynamics, vol. 70, no. 1, pp. 619-625, 2012. 


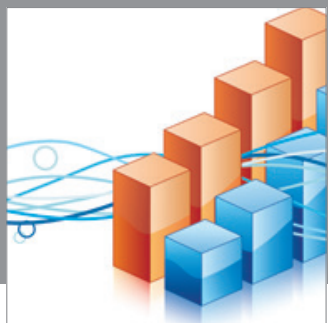

Advances in

Operations Research

mansans

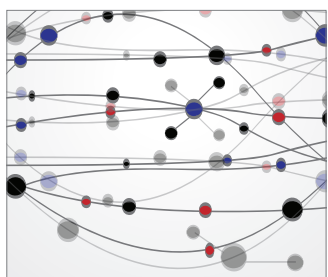

The Scientific World Journal
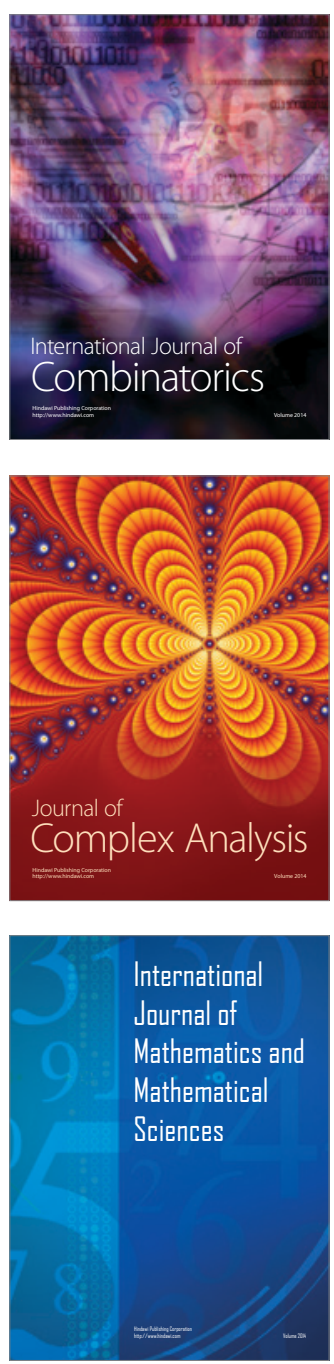
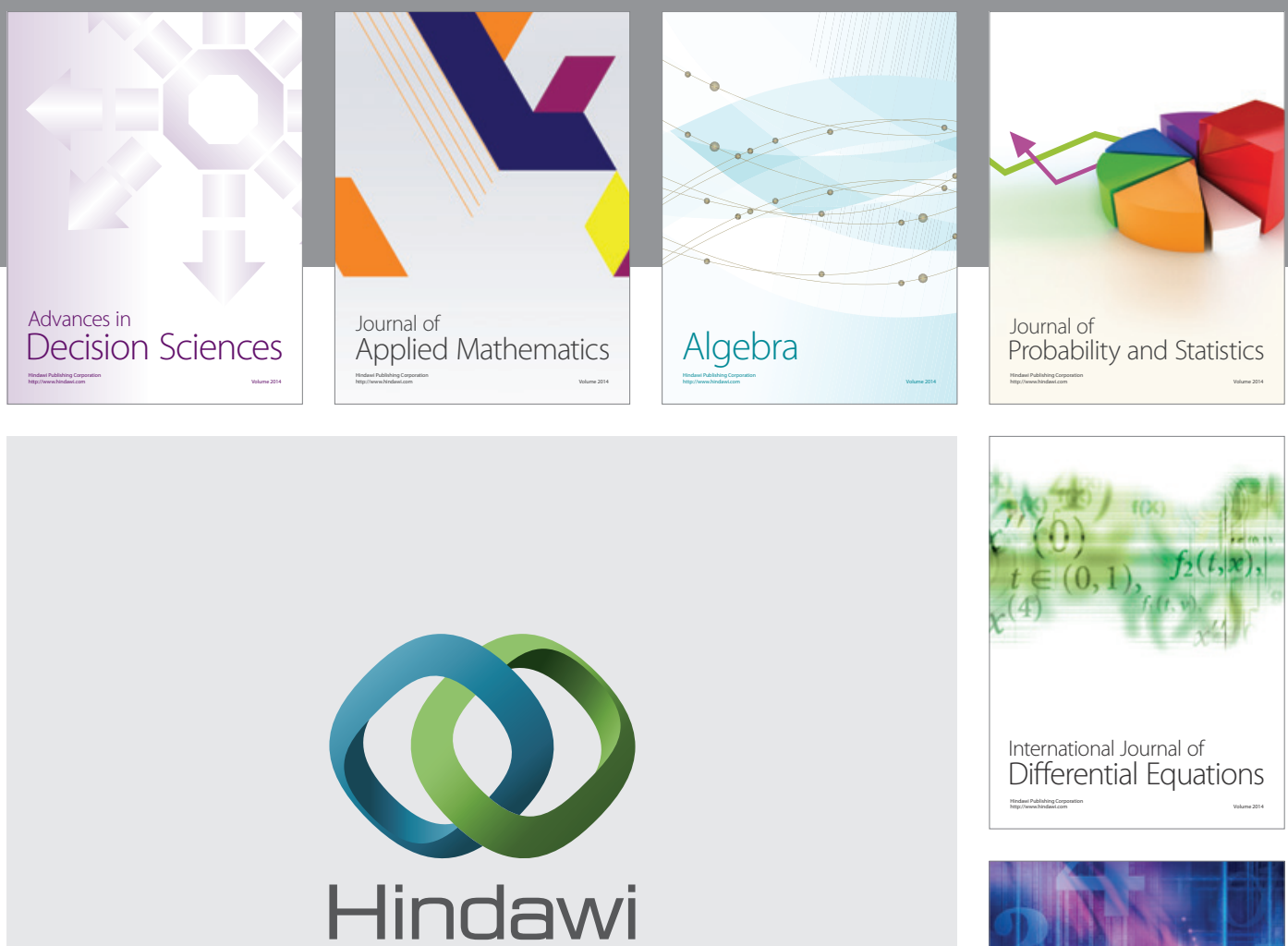

Submit your manuscripts at http://www.hindawi.com
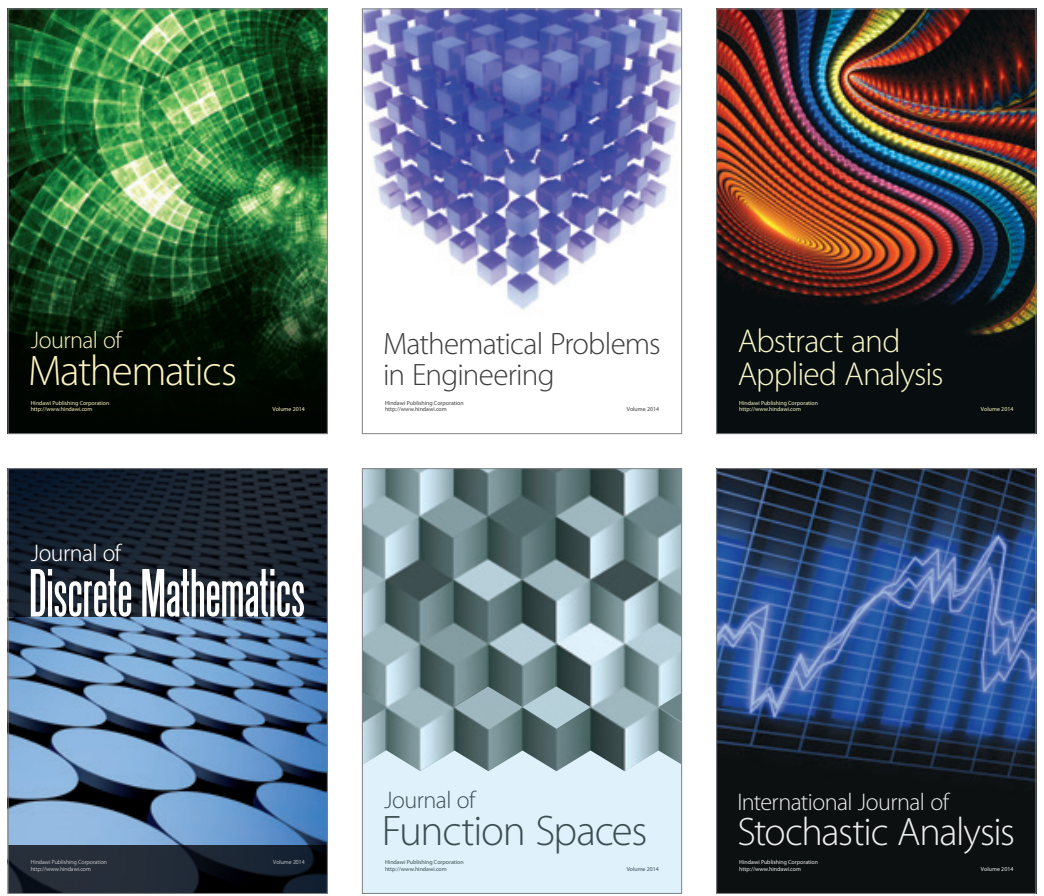

Journal of

Function Spaces

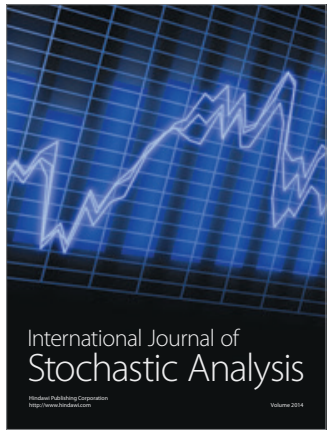

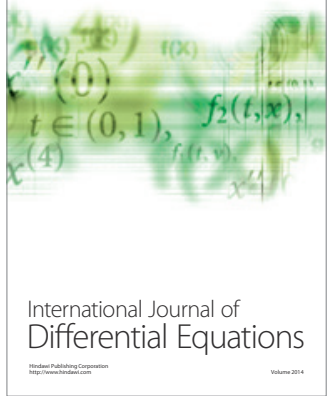
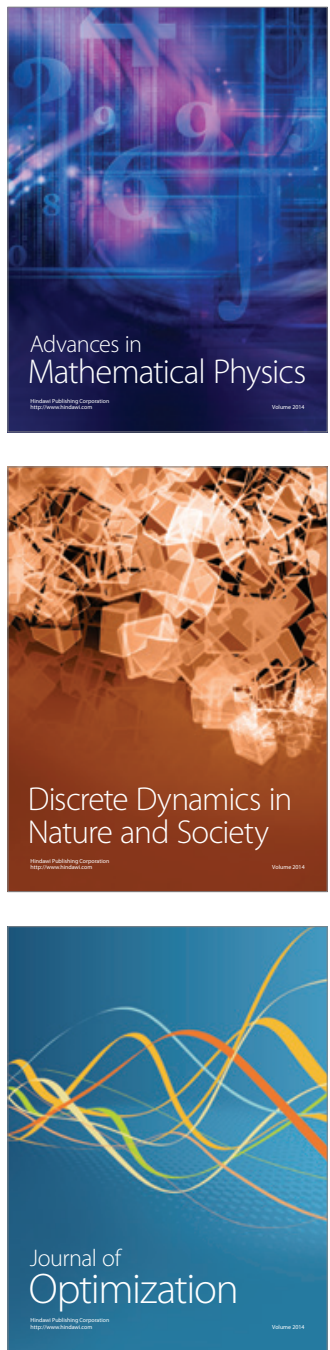